\title{
Effectiveness and safety of polymyxin B for the treatment of infections caused by extensively drug-resistant Gram-negative bacteria in Thailand
}

This article was published in the following Dove Press journal: Infection and Drug Resistance

\author{
Thundon Ngamprasertchai' \\ Adhiratha Boonyasiri ${ }^{2}$ \\ Lantharita Charoenpong ${ }^{3}$ \\ Sireethorn Nimitvilai ${ }^{4}$ \\ Narisorn Lorchirachoonkul ${ }^{5}$ \\ Luksame \\ Wattanamongkonsil ${ }^{2}$ \\ Visanu Thamlikitkul ${ }^{6}$ \\ 'Department of Clinical Tropical \\ Medicine, Faculty of Tropical \\ Medicine, Mahidol University, \\ Bangkok, Thailand; '2Department \\ of Research and Development, \\ Faculty of Medicine Siriraj Hospital, \\ Mahidol University, Bangkok, \\ Thailand; ${ }^{3}$ Division of Medicine, \\ Chaophraya Yommarat Hospital, \\ Suphanburi, Thailand; ${ }^{4}$ Division of \\ Medicine, Nakhornpathom Hospital, \\ Nakhornpathom, Thailand; ${ }^{5}$ Division \\ of Medicine, Ratchaburi Hospital, \\ Ratchaburi, Thailand; 'Department of \\ Medicine, Faculty of Medicine Siriraj \\ Hospital, Mahidol University, Bangkok, \\ Thailand
}

Background: Colistimethate sodium (colistin) has been used in the treatment of infections caused by extensively drug-resistant (XDR) Gram-negative bacteria in Thailand over the past decade, with a mortality rate of $50 \%$ and a nephrotoxicity rate of $40 \%$. Polymyxin $\mathrm{B}$ has not been available in Thailand. We conducted a Phase II clinical study to determine the effectiveness and safety of polymyxin B, compared with colistin, for the treatment of XDR Gram-negative bacterial infections in Thai patients.

Methods: A total of 73 adult patients hospitalized at four participating tertiary care hospitals from January 2015 to December 2015 who had infections caused by XDR Gram-negative bacteria and had to receive colistin were enrolled in the study. Polymyxin B (100 mg/day) was administered intravenously every 12 hours for 7-14 days.

Results: Most of the patients were older males with comorbidities who had received antibiotics, particularly carbapenems, prior to receiving polymyxin B. More than half of the patients had pneumonia, and $51.5 \%$ of the infections were caused by XDR Acinetobacter baumannii, which was susceptible to colistin. Good clinical responses at the end of treatment were observed in $78.1 \%$ of cases, the overall 28 -day mortality rate from all causes was $28.7 \%$, the microbiological clearance of the targeted bacteria after therapy was $56.2 \%$ and nephrotoxicity occurred in $24.7 \%$ of cases. Neurotoxicity relating to reversible numbness was observed in two cases.

Conclusion: Polymyxin B seems to be effective and safe for the treatment of XDR Gramnegative bacterial infections. Polymyxin B should be considered as an alternative to colistin for treatment of infections caused by XDR Gram-negative bacteria in Thai adult patients, especially those at risk of nephrotoxicity.

Keywords: mortality, acute kidney injury, Acinetobacter baumannii

\section{Background}

Hospital-acquired infections (HAIs) are prevalent in Thailand, and Gram-negative bacteria are the predominant etiologic agents. ${ }^{1}$ In Thailand, HAIs are responsible for at least 38,000 deaths and an additional 3.2 million hospitalization days annually, with an economic burden of $\sim 1 \%$ of gross domestic products. ${ }^{2}$ Acinetobacter baumannii is the leading cause of drug-resistant HAIs. ${ }^{2}$ Along with A. baumannii, many other Gram-negative bacterial isolates causing HAIs, such as Pseudomonas aeruginosa, have been found to be extensively drug resistant (XDR) (i.e., are susceptible to only one or two categories of antimicrobial agents). ${ }^{3}$ However, most XDR Gram-negative bacteria remain susceptible to the traditional antibiotics polymyxins.

Polymyxins have been reintroduced as the last line of defense against XDR Gramnegative bacterial infections over the past decade. ${ }^{4}$ Parenteral polymyxin E or colistin
Correspondence: Thundon Ngamprasertchai

Department of Clinical Tropical Medicine, Faculty of Tropical Medicine, Mahidol University 420/6 Ratchawithi Road, Ratchathewi, Bangkok 10400, Thailand Tel/fax +66 235491004 Email thundon_ngamprasertchai@ hotmail.com 
has been used in the form of colistimethate sodium (CMS), an inactive prodrug of colistin that requires conversion to its active form after administration. ${ }^{4}$ The effectiveness of CMS for XDR Gram-negative infections was modest with a cure rate of $30 \%-75 \%$, an overall mortality rate of $15 \%-60 \%$ and nephrotoxicity occurring in $10 \%-60 \%$ of cases. ${ }^{5-7}$

Parenteral polymyxin B has several advantages over colistin. ${ }^{4}$ It is an active drug, and a loading dose may therefore not be necessary. It is excreted by non-renal clearance, meaning that dose adjustment in patients with impaired renal function is not generally needed. Polymyxin B showed good in vitro activity against $A$. baumannii isolated from Thai patients and displayed greater activity than colistin. ${ }^{8}$ Several studies on the effectiveness and safety of parenteral polymyxin B for the treatment of resistant Gram-negative bacterial infections have reported that polymyxin $\mathrm{B}$ is effective and safe. ${ }^{9-12}$ However, parenteral polymyxin B is not available in Thailand, and its effectiveness and safety for treatment of Gram-negative infections in Thai patients are unknown. Therefore, the aim of this study was to determine the effectiveness and safety of parenteral polymyxin B for the treatment of XDR Gramnegative bacterial infections in Thai patients.

\section{Methods}

This Phase II clinical study was conducted in four tertiary care hospitals, namely, Siriraj Hospital, Chaophraya Yommarat Hospital, Nakhornpathom Hospital and Ratchaburi Hospital, from January 2015 to December 2015. The study protocol was approved by the Siriraj Institutional Review Board no. 568/2557 and the ethics committee on human research of the other respective hospitals. All enrolled patients or their legal representatives signed informed consent forms before participating in the study.

We performed Gram-negative bacteria antimicrobial susceptibility test consisting of aminoglycosides, cephalosporins, fluoroquinolones, carbapenems, tetracycline, beta-lactam/beta-lactamase inhibitors, trimethoprim/sulfamethoxazole and polymyxins. XDR Gram-negative bacteria refer to bacteria that are susceptible to only one or two categories of antimicrobial agents, which usually are polymyxins.

Eligible patients were adults aged from 18 to 80 years who had infections caused by XDR Gram-negative bacteria requiring polymyxin treatment for $\leq 14$ days, who also displayed serum creatinine levels $<3 \mathrm{mg} / \mathrm{dL}$ or a glomerular filtration rate $(\mathrm{GFR}) \geq 15 \mathrm{~mL} / \mathrm{min} / 1.73 \mathrm{~m}^{2}$, had a life expectancy $>48$ hours and had or had not received colistin $<48$ hours prior to the study. The exclusion criteria encompassed pregnant or lactating women, patients with a body mass index (BMI) of $>30 \mathrm{~kg} / \mathrm{m}^{2}$, patients who had concurrent neuromuscular disease, those who had received renal replacement therapy and those with a known allergy to polymyxins.

Each subject received intravenous polymyxin B (Polymyxin B for Injection USP; X-Gen Pharmaceuticals, Inc., Horseheads, NY, USA) at $2 \mathrm{mg} / \mathrm{kg} /$ day for 7-14 days. Concomitant antibiotic(s) were given at the discretion of the attending physician. The enrolled subjects were assessed for clinical outcomes 28 days after initiating polymyxin B therapy and at the end of treatment. Microbiological outcomes were assessed on Day 3 and at the end of polymyxin B therapy. Acute kidney injury was assessed according to the RIFLE criteria as risk (serum creatinine increased 1.5 times above baseline or GFR decreased $>25 \%$ ), injury (serum creatinine increased two times above baseline or GFR decreased $>50 \%$ ), failure (serum creatinine increased three times above baseline or GFR decreased 75\%), loss of kidney function (complete loss of renal function for $>4$ weeks) or end-stage kidney disease (complete loss of renal function for $>3$ months). ${ }^{13}$ Neurological toxicity was determined by clinical examination.

Clinical outcomes were categorized as a good response, failure, relapse or death. A good clinical response was defined as the absence of, or improvement in, the attributable signs and symptoms of the target infection; failure was defined as clinical deterioration of the target infection after 3 days of polymyxin B therapy; relapse was defined as an infection with the same target bacteria after displaying a good clinical response to polymyxin B. Microbiological outcomes were categorized as eradication of the target bacteria, persistence of the target bacteria or super-infection with other bacteria.

It was estimated that the effectiveness of polymyxin B for the treatment of XDR Gram-negative bacterial infections in terms of overall 28-day mortality after receiving polymyxin B was $25 \% \pm 10 \%$ with $5 \%$ type I error (two sided). Therefore, 73 eligible patients were needed. Categorical data were summarized by frequency, whereas continuous data were summarized by the mean, median, standard deviation (SD) and range as appropriate.

\section{Results}

A total of 73 patients who received polymyxin B for $>48$ hours for the treatment of XDR Gram-negative bacterial infections were included in this study. The baseline characteristics of the patients are given in Table 1 . The mean age (SD) of the patients was 59.8 years (15.6 years), and $54.8 \%$ were males. Half of the patients had a history of hospitalization in the preceding 90 days. The mean BMI of the patients was $21.3 \mathrm{~kg} / \mathrm{m}^{2}$. Most of the patients were hospitalized at 
Table I Baseline characteristics of the 73 patients

\begin{tabular}{|c|c|}
\hline Characteristics & Findings \\
\hline Male, $n(\%)$ & $40(54.8)$ \\
\hline Mean age (years), mean \pm SD (range) & $59.8 \pm 15.6(20-80)$ \\
\hline Mean BMI $\left(\mathrm{kg} / \mathrm{m}^{2}\right)$, mean \pm SD (range) & $21.3 \pm 4(13-30)$ \\
\hline Previous hospitalization within the past 3 months, $\mathbf{n}(\%)$ & $37(50.7)$ \\
\hline \multicolumn{2}{|l|}{ Study sites, n (\%) } \\
\hline Siriraj Hospital & $42(57.5)$ \\
\hline Chaophraya Yommarat Hospital & $18(24.7)$ \\
\hline Nakhornpathom Hospital & II (I5) \\
\hline Ratchaburi Hospital & $2(2.7)$ \\
\hline Underlying conditions, $\mathbf{n}(\%)$ & $69(94.5)$ \\
\hline Diabetes mellitus with or without other medical conditions & $21(28.8)$ \\
\hline Cerebrovascular diseases & $17(23.6)$ \\
\hline Cardiovascular diseases & $10(13.9)$ \\
\hline Solid malignancy & $10(13.9)$ \\
\hline Hematologic malignancy & $9(12.5)$ \\
\hline Chronic liver diseases & $8(11.6)$ \\
\hline Chronic kidney diseases & $6(8.3)$ \\
\hline Previous exposure to antibacterial agents in the preceding 14 days, $n(\%)$ & $70(95.9)$ \\
\hline Carbapenems & $53(40.2)$ \\
\hline Beta-lactam/beta-lactamase inhibitor & $26(19.7)$ \\
\hline Vancomycin & $16(12.1)$ \\
\hline Ceftriaxone & $16(12.1)$ \\
\hline \multicolumn{2}{|l|}{ Locations of hospitalization, $\mathrm{n}(\%)$} \\
\hline Medicine ICU & $29(39.7)$ \\
\hline Surgery ICU & $12(16.4)$ \\
\hline Medicine & $24(32.9)$ \\
\hline Surgery & $8(\mathrm{II})$ \\
\hline
\end{tabular}

Abbreviations: SD, standard deviation; BMI, body mass index; ICU, intensive care unit.

Siriraj Hospital and had underlying diseases, with diabetes mellitus being the most common. Nearly all of the patients had received antibacterial agents, including carbapenems, in the 14 days preceding the study.

The clinical characteristics of the patients are given in Table 2. The mean Acute Physiology and Chronic Health Evaluation II (APACHE II) value and the mean length of hospital stay were 19.5 and 42.3 days, respectively. A high number of patients $(80.8 \%)$ received mechanical ventilation. The patients received intravenous polymyxin $\mathrm{B}$ at a mean dosage of $1.9 \mathrm{mg} / \mathrm{kg} /$ day with a mean (range) duration of treatment of 11.7 days (4-14 days). Pneumonia was the most common type of infection (60.3\%), followed by tracheobronchitis (20.5\%) and bloodstream infections (15.1\%). A. baumannii was the most common causative bacteria $(51.5 \%)$, and all strains were susceptible to colistin. Other causative bacteria were $P$. aeruginosa $(24.6 \%)$, Klebsiella pneumoniae (8.2\%), methicillin-resistant Staphylococcus aureus (4.5\%) and Stenotrophomonas maltophilia (3\%). Additional antibiotics were administered to $72.6 \%$ of the patients including carbapenems $(22.5 \%)$, levofloxacin (14.1\%) and vancomycin (12.7\%).

The treatment outcomes are given in Table 3. A good clinical response at the end of treatment was observed in $78.1 \%$ of the patients. The overall 28 -day mortality rate from all causes was $28.7 \%$. Microbiological eradication of the target bacteria at the end of therapy occurred in $56.2 \%$ of patients. Nephrotoxicity was observed in 18 (24.7\%) patients, four $(5.5 \%)$ of whom required renal replacement. Only two $(2.8 \%)$ patients reported reversible numbness of extremities.

\section{Discussion}

Colistin is available in some countries, whereas polymyxin $\mathrm{B}$ is not available in many of these countries, including Thailand. Colistin has been used for therapy of infections due to XDR Gram-negative bacteria in Thailand over the past 12 years with modest effectiveness and frequent nephrotoxicity. Therefore, we would like to determine if polymyxin B, which is not available in Thailand, could be an alternative to colistin in terms of non-inferior effectiveness and less 
Table 2 Clinical characteristics of the 73 patients

\begin{tabular}{|c|c|}
\hline Characteristics & Findings \\
\hline APACHE II, mean \pm SD (range) & $19.5 \pm 6.9(2-42)$ \\
\hline Length of hospital stay (days), mean \pm SD (range) & $42.3 \pm 27(8-144)$ \\
\hline Mechanical ventilation, $\mathrm{n}(\%)$ & $59(80.8)$ \\
\hline Polymyxin B dosage $(\mathrm{mg} / \mathrm{kg} /$ day $)$, mean \pm SD (range) & $1.9 \pm 0.3(1.27-2.63)$ \\
\hline Treatment duration (days), mean \pm SD (range) & $11.7 \pm 3.2(4-14)$ \\
\hline \multicolumn{2}{|l|}{ Type of infection, $n(\%)$} \\
\hline Pneumonia & $44(60.3)$ \\
\hline Tracheobronchitis & $15(20.5)$ \\
\hline Bloodstream infection & II (I5.I) \\
\hline Intra-abdominal infection & $3(4.1)$ \\
\hline Urinary tract infection & $2(2.7)$ \\
\hline Skin and soft tissue infection & $2(2.7)$ \\
\hline \multicolumn{2}{|l|}{ Causative bacteria, $\mathbf{n}(\%)$} \\
\hline Acinetobacter baumannii & $69(51.5)$ \\
\hline Pseudomonas aeruginosa & $33(24.6)$ \\
\hline Klebsiella pneumoniae & II (8.2) \\
\hline MRSA & $6(4.5)$ \\
\hline Stenotrophomonas maltophilia & $4(3)$ \\
\hline Concomitant antimicrobial agents ${ }^{\mathrm{a}}, \mathrm{n}(\%)$ & $53(72.6)$ \\
\hline Carbapenems & $16(22.5)$ \\
\hline Levofloxacin & $10(14.1)$ \\
\hline Vancomycin & $9(12.7)$ \\
\hline Antifungal agents & $7(9.9)$ \\
\hline Tigecycline & $5(7)$ \\
\hline Cefoperazone-sulbactam & $5(7)$ \\
\hline Metronidazole & $5(7)$ \\
\hline
\end{tabular}

Note: aPatients who received more than one agent.

Abbreviations: APACHE II, Acute Physiology and Chronic Health Evaluation II; SD, standard deviation; MRSA, methicillin-resistant Staphylococcus aureus.

Table 3 Treatment outcomes of the 73 patients

\begin{tabular}{ll}
\hline Outcomes & $\mathbf{n}(\%)$ \\
\hline Good clinical response at the end of polymyxin B treatment & $57(78.1)$ \\
28-day mortality rate from all causes & $21(28.7)$ \\
Microbiological clearance at the end of therapy & $4 I(56.2)$ \\
Nephrotoxicity & $18(24.7)$ \\
Risk & $8(11)$ \\
Injury & $6(8.2)$ \\
Failure & $4(5.5)$ \\
Neurotoxicity & $2(2.8)$ \\
\hline
\end{tabular}

nephrotoxicity. The present study is a Phase II study of a new drug name polymyxin $\mathrm{B}$ that determines if polymyxin $\mathrm{B}$ could be a potential effective and safe antibiotic for therapy of infections due to XDR Gram-negative bacteria in Thai patients. Therefore, only one group of the study patients was included. It will be unethical to have a control group of the patients infected with XDR Gram-negative bacteria who received carbapenems or other antibiotics that the isolated organisms were resistant to. Having the control group with colistin is not feasible since its efficacy would be similar to that of polymyxin B and a very large sample size will be needed. The effectiveness and safety of polymyxin B for the treatment of XDR Gram-negative infections observed in this study were comparable to the results of previous studies. ${ }^{9-12}$ The dosing regimen for polymyxin B used in this study was based on the manufacturer's recommendations. It is possible that the effectiveness of polymyxin $B$ could be improved by optimizing the dosing regimen for the target patients. A population pharmacokinetic study of polymyxin B in Thai patients is currently being conducted to determine the most appropriate dosing regimen for Thai patients. The rate of good response to polymyxin $\mathrm{B}$ observed in this study should not related to the prior use of carbapenems or a combination of polymyxin B and carbapenems because all isolated bacteria from the patients were resistant to carbapenems and the results from the recent randomized controlled trial (RCT) revealed that the outcomes of the patients infected with XDR Gram-negative bacteria who received a combination of colistin and meropenem was not significantly better than those who received colistin alone. ${ }^{14}$ Regarding the nephrotoxicity of polymyxin $\mathrm{B}$, the findings from our study were in accor- 
dance with the recent reviews reporting that polymyxin B was associated with less risk of nephrotoxicity than colistin..$^{15,16}$

To our knowledge, no previous studies have reported a direct comparison of the effectiveness and safety of polymyxin B with colistin for the treatment of XDR Gramnegative infections. A recent report that analyzed 138 hospitalized adult patients at Siriraj Hospital with documented Gram-negative bacterial infections from October 2014 to June 2015, who had received colistin for $>48$ hours, revealed favorable clinical outcomes in $71.7 \%$ of the patients at the end of colistin therapy compared with mortality rates of $23.2 \%$ after 30 days and $39.9 \%$ at the end of therapy. ${ }^{17}$ The rate of microbiological eradication of the target bacteria at the end of colistin therapy was found to be $50.0 \%$, and the overall incidence of acute kidney injury was $39.9 \% .{ }^{17}$ Based on the findings of the present study, the effectiveness and safety of polymyxin B for the treatment of XDR Gram-negative infections appear higher than those of colistin. Polymyxin B is associated with an improved clinical response at the end of treatment, increased microbiological eradication, lower overall mortality and a lower incidence of nephrotoxicity compared with colistin. ${ }^{17,18}$ Mild reversible neurotoxicity was rare in the present study and was not observed in the previous report. ${ }^{17}$ However, a randomized controlled trial of polymyxin $B$ versus colistin is now needed to confirm whether polymyxin B is more effective and safer than colistin. It should be noted that the polymyxin $\mathrm{B}$ concentration in urine is less than that of colistin, ${ }^{4}$ so colistin should be used in favor of polymyxin $B$ to treat urinary tract infections. However, some patients in the present study had urinary tract infections with bacteremia, and all showed a good clinical response after treatment with polymyxin $\mathrm{B}$.

This study was the first clinical study in Thai patients to determine the effectiveness and safety of parenteral polymyxin B for the treatment of XDR Gram-negative infections. The study sites included university hospitals and other tertiary care hospitals, and the enrolled patients had infections at various sites. All of these factors helped to ensure that the findings were generally applicable to Thai patients with XDR Gram-negative infections.

This study had several limitations. First, it was a clinical trial that lacked an appropriate control group for comparison. Second, we excluded some patients who had received renal replacement therapy or suffered from obesity (BMI of $>30 \mathrm{~kg} / \mathrm{m}^{2}$ ). Third, since the microbiology laboratory did not routinely perform polymyxin B susceptibility testing, the colistin susceptibility test results had to be used instead. However, a previous report revealed that polymyxin B was more active than colistin, ${ }^{8}$ and for this reason, the bacteria isolated from the patients in the present study were kept for future studies on polymyxin B susceptibility. Although this study is Phase II clinical study without the concurrent control group, the results from this study confirm the effectiveness and safety of polymyxin B. Such information will be valuable for the responsible institute and Thailand Food and Drug Administration to consider importing and/or producing polymyxin B for therapy of XDR Gram-negative bacterial infections in Thai patients. Moreover, such information may be useful for the countries with availability of only colistin to consider if polymyxin B should also be available in addition to colistin.

\section{Conclusion}

Polymyxin B appears to be effective and safe for the treatment of infections caused by XDR Gram-negative bacteria in Thai adult patients. Nephrotoxicity related to polymyxin B exposure seems to be less common than with colistin based on the present study and previous studies of Thai patients. Polymyxin B should therefore be considered as an effective alternative therapy for infections caused by XDR Gram-negative bacteria, especially in patients at risk of nephrotoxicity.

\section{Acknowledgments}

The authors acknowledge the support of Ms. Sasima Tongsai for statistical analysis of the data and Ms. Sukonthip Popum for coordination of the study. This study was presented as a poster presentation in 26th European Congress of Clinical Microbiology and Infectious Diseases (ECCMID), which took place in Amsterdam, Netherland, 2016. This study was funded by the Health Systems Research and Development Project, the Faculty of Medicine Siriraj Hospital, the Thai Health Promotion Fund, the Health Systems Research Institute (Thailand) and the Government Pharmaceutical Organization.

\section{Disclosure}

The authors report no conflicts of interest in this work.

\section{References}

1. Rongrungruang Y, Sawanpanyalert N, Chomdacha P, et al. Healthcare associated infections in Thailand 2011. J Med Assoc Thai. 2013;96(Suppl 2):S117-S123.

2. Phumart P, Phodha T, Thamlikitkul V, Riewpaiboon A, Prakongsai $\mathrm{P}$, Limwattananon S. Health and economic impacts of antimicrobial resistant infections in Thailand: a preliminary study. $J$ Health Syst Res. 2012;3(6):352-360 
3. Magiorakos AP, Srinivasan A, Carey RB, et al. Multidrug-resistant, extensively drug-resistant and pandrug-resistant bacteria: an international expert proposal for interim standard definitions for acquired resistance. Clin Microbiol Infect. 2012;18(3):268-281.

4. Nation RL, Velkov T, Li J. Colistin and polymyxin B: peas in a pod, or chalk and cheese? Clin Infect Dis. 2014;59(1):88-94.

5. Zhang H, Zhang Q. Clinical efficacy and safety of colistin treatment in patients with pulmonary infection caused by Pseudomonas aeruginosa or Acinetobacter baumannii: a meta-analysis. Arch Med Sci. 2015;11(1):34-42.

6. Chen Z, Chen Y, Fang Y, et al. Meta-analysis of colistin for the treatment of Acinetobacter baumannii infection. Sci Rep. 2015;5:17091.

7. Gu WJ, Wang F, Tang L, Bakker J, Liu JC. Colistin for the treatment of ventilator-associated pneumonia caused by multidrug-resistant Gram-negative bacteria: a systematic review and meta-analysis. Int $J$ Antimicrob Agents. 2014;44(6):477-485.

8. Thamlikitkul V, Tiengrim S, Seenama C. In vitro activity of polymyxin B against carbapenem-resistant Acinetobacter baumannii. J Med Assoc Thai. 2014;97(12):1254-1258.

9. Ouderkirk JP, Nord JA, Turett GS, Kislak JW. Polymyxin B nephrotoxicity and efficacy against nosocomial infections caused by multiresistant gramnegative bacteria. Antimicrob Agents Chemother. 2003;47(8):2659-2662.

10. Crusio R, Rao S, Changawala N, et al. Epidemiology and outcome of infections with carbapenem-resistant Gram-negative bacteria treated with polymyxin B-based combination therapy. Scand J Infect Dis. 2014;46(1):1-8.

11. Rigatto MH, Ribeiro VB, Konzen D, Zavascki AP. Comparison of polymyxin B with other antimicrobials in the treatment of ventilatorassociated pneumonia and tracheobronchitis caused by Pseudomonas aeruginosa or Acinetobacter baumannii. Infection . 2013;41(2):321-328.
12. Nelson BC, Eiras DP, Gomez-Simmonds A, et al. Clinical outcomes associated with polymyxin B dose in patients with bloodstream infections due to carbapenem-resistant Gram-negative rods. Antimicrob Agents Chemother. 2015;59(11):7000-7006.

13. Bellomo R, Ronco C, Kellum JA, Mehta RL, Palevsky P; Acute Dialysis Quality Initiative Workgroup. Acute renal failure-definition, outcome measures, animal models, fluid therapy and information technology needs: the Second International Consensus Conference of the Acute Dialysis Quality Initiative (ADQI) Group. Crit Care. 2004;8(4):R204-R212.

14. Paul M, Daikos GL, Durante-Mangoni E, et al. Colistin alone versus colistin plus meropenem for treatment of severe infections caused by carbapenem-resistant Gram-negative bacteria: an open-label, randomised controlled trial. Lancet Infect Dis. 2018;18(4):391-400.

15. Vardakas KZ, Falagas ME. Colistin versus polymyxin B for the treatment of patients with multidrug-resistant Gram-negative infections: a systematic review and meta-analysis. Int $J$ Antimicrob Agents. 2017;49(2):233-238

16. Zavascki AP, Nation RL. Nephrotoxicity of polymyxins: is there any difference between colistimethate and polymyxin B? Antimicrob Agents Chemother. 2017;61(3):e2319-e2316.

17. Thamlikitkul V, Popum S. Monitoring of effectiveness and safety of colistin for therapy of resistant Gram negative bacterial infections in hospitalized patients at Siriraj hospital. J Med Assoc Thai. 2016;99(3):301-307.

18. Rigatto MH, Oliveira MS, Perdigão-Neto LV, et al. Multicenter prospective cohort study of renal failure in patients treated with colistin versus polymyxin B. Antimicrob Agents Chemother. 2016;60(4): 2443-2449.
Infection and Drug Resistance

\section{Publish your work in this journal}

Infection and Drug Resistance is an international, peer-reviewed openaccess journal that focuses on the optimal treatment of infection (bacterial, fungal and viral) and the development and institution of preventive strategies to minimize the development and spread of resistance. The journal is specifically concerned with the epidemiology of antibiotic

\section{Dovepress}

resistance and the mechanisms of resistance development and diffusion in both hospitals and the community. The manuscript management system is completely online and includes a very quick and fair peerreview system, which is all easy to use. Visit http://www.dovepress.com/ testimonials.php to read real quotes from published authors. 